\title{
Classifying Functioning of Children and Adolescents with Intellectual Disability: The Utility of the International Classification of Functioning, Disability and Health for Children and Youth
}

Maria do Carmo Vale, Lu's Pereira-da-Silva, Maria Joao Pimentel, Teresa Nunes Marques, Helena Rodrigues $^{\S}$, Gilda Cunha, Maria do C'eu Machado, Manuela Sanches-Ferreira, and Rune J. Simeonsson

\section{Abstract}

Observational cross-sectional study carried out in a pediatric neurodevelopment unit of a tertiarycare hospital. A sample of 355 chil- dren with median ((min.-max.) 1.0-17.3) years with intellectual disability (30.4\% borderline, $43.1 \%$ mild, $19.7 \%$ moderate, $5.1 \%$ severe, and $1.7 \%$ profound disability) was seen over a period of 3 years. Based on clinical observation and psychological evaluation, a neurodevelopmental pediatrician selected ICF-CY body functions codes, and respective qualifiers, to effectively describe functioning of children. Based on evaluation reports, a psychologist and a special educator assigned the previously chosen ICF-CY body functions codes to 139 and 67 children, respectively. Inter-rater agreement was estimated using simple and weighted Cohen's kappa coefficients and Gwet's AC1 statistic and Gwet's weighted kappa coefficient statistic. A set of eight ICF-CY codes was identified as efficiently describing impairments of body functions of children with intellectual disability: global mental functions b117 and b122; specific mental functions b147, b163, b164, and b167; and voice and speech functions b320 and b330. Results indicate a correspondence between the level of severity of qualifiers assigned to ICF-CY codes and the level of intellectual disability. Inter-rater agreement was variable among raters, with the best agreements found for qualifying intellectual functions (b117) and psychomotor functions (b122). A profile of eight ICF-CY codes effectively describes functioning of children with intellectual disability, providing an alterna- tive to medically based classification, based on diagnoses with functionally based classification of children's characteristics. The find- ings contribute to define a comprehensive set of codes to reliably record individual differences of functioning in this population.

Keywords: Disability and Health for Children and Youth (ICF-CY), International Classification of Functioning, intellectual dis- abilities, interobserver agreement 


\section{Introduction}

Historically, defining physical and mental disability was based on a medical approach, by focusing on etiology and assignment of diagnoses using the International Classification of Diseases (ICD-

10) (WHO, 2007a) and the Diagnostic and Statistical Manual of Mental Disorders (DSM-5) (American Psychiatric Association, 2013). The end of the 20th century marked a paradigm shift from medically based models of disability to multidimensional, compre- hensive models based on health and functioning. This shift was reflected in the adoption of new policies and practices, and in the publication of the $U$. N. Convention on the Rights of Persons with Disabilities (UNCRPD, 2007) and the International Classification of Functioning, Disability and Health - ICF (WHO, 2001). As uni- versal documents, UNCRPD offered definitions of rights within an inclusive framework of human functioning and participation and the ICF provided a biopsychosocial model for their classification.

The ICF and specifically its expanded version for Children and Youth (ICF-CY) provide a comprehensive approach to classi- fy dimensions of disability in children and adolescents (WHO, 2007a) by conceptualizing human functioning and disability as a product of the interaction between the person and the environ- ment in which she functions (Lee, 2011; Simeonsson, 2006, 2009). Within this context, the ICF-CY provides taxonomy orga- nized into four main components-body functions, body struc- tures, activities and participation, and environmental factors - covering different dimensions of functioning and health. With content defined in a neutral language, the ICF-CY offers docu- mentation of functioning in each component through a list of alphanumeric codes-with the letter corresponding to the com- ponent $(b$ for body functions, $s$ for body structures, $d$ for activi- ties and participation, $e$ for environmental factors) followed by a number to identify the domain to which the code belongs (e.g., b140: mental function of attention). The assignment of a qualifier to the code allows identification of the extent or severity of dis- turbances in body function impairments, activities and participa- tion limitations and restrictions and environmental barriers or facilitators. Qualifier values defining the severity of problems range from 1 (mild) to 4 (complete), the absence of a problem can be documented with the qualifier 0 (no problem). The quali- fiers 8 or 9 are used to indicate that the extent of severity of a code cannot be specified or is not applicable, respectively. Given its developmental focus, the ICF-CY can serve as a common lan- guage for professionals in education, health, and social work to describe the functioning of children and adults with disabilities (Simeonsson, Simeonsson, \& Hollenweger, 2008).

A central contribution of the functional approach of the ICF- CY is that it offers a comprehensive and systematic basis for assessment of students' support needs beyond that of diagnoses (Lollar \& Simeonsson, 2005). In this context, the ICF-CY pro- vides the basis for classifying the nature and severity of functional limitations, yielding a profile of individual differences (Sanches- Ferreira et al., 2013). In keeping with the new paradigm of human functioning and disability, the ICF-CY is increasingly being 
used as a universal language to describe and classify func- tioning in children and adolescents across health, education, and disciplinary fields (Peterson, Simeonsson, Enskar, \& Huus, 2013). In this regard, the ICF-CY has been applied in mapping child- ren's health data on a population level (Stahl, Granlund, Gare- Andersson, \& Enskar, 2011) and proposed as a comprehensive tool for research, clinical practice, and quality management (Raghavendra, Bornman, Granlund, \&Bj€rck-Akesson, 2007).

It is also applied in national policy, for example, in Taiwan where it serves as the basis for assessment to determine eligibility for disability services (Huang et al., 2015). In Portugal, the ICF- CY framework is of compulsory use as the base for assessment and eligibility processes of children for special education services. The Portuguese special education law states that the ICF-CY- based assessment must form the basis for developing the child's functioning profile. In turn, the nature and severity of impair- ments of body functions and structures and their impact on the child's activities and participation-as described in the function- ing profile-serve as the basis for determining the child's eligibility for special education and habilitation (Direçao Geral de Inovaçao e Desenvolvimento Curricular, 2010). This application supports collaboration between educational and health professionals involved in the specialized assessment conducted in reference to use of the ICF-CY.

A challenge to collaboration documentation of childhood dis- ability is the lack of a coherent and universal approach for assign- ing diagnoses resulting in inconsistencies in the determination of children's eligibility for services. In particular, inconsistencies may be due to lack of correspondence between diagnoses and the learn- ing and social problems faced by students (Sanches-Ferreira et al., 2013). A second challenge pertains to establishing observer agree- ment among care professionals representing different disciplinary backgrounds and different forms and levels of training. The range of professionals involved in the care of children and adolescents include doctors, psychologists, occupational therapists, teachers, physiotherapists, social workers, and public health and public pol- icy makers (Raghavendra et al., 2007). Variability in observer agreement could be due to the fact that some professionals may rely on direct observation and clinical judgment in classification, whereas others may rely on evaluation reports and data.

Interobserver agreement in using the ICF among different professionals in different scenarios represents a continuing prior- ity for research. The WHO encourages calibrating reliability of ICF terminology—codes and qualifiers—against existing mea- sures (WHO, 2001; Grill, Mansmann, Cieza, \& Stucki, 2007). Some studies have assessed interobserver agreement using the ICF-CY in describing and classifying functioning in children and adolescents with special health care (Kronk, Ogonowski, Rice, \& Feldman, 2005; Grill et al., 2007; Walsh et al., 2014). Sufficient reliability when observing the same child attribute with the same scale is dependent on several factors, including operationalization of the concepts to be measured, the performance of the child, and different evaluation capacity of different observers (Grill et al., 2007).

In this study, we hypothesize significant reliability among professionals in applying a set of ICF-CY codes to profile individ- ual differences in severity of functioning of children with intellec- tual disabilities. Profiling functional characteristics of the basis of severity is particularly important for children in the 
borderline range of intellectual functioning who are not identified in current diagnostic classifications (ICD-10; DSM 5), and consequently may not be eligible for needed educational and social support. As such, the standard language of codes in the ICF-CY can contrib- ute to evaluation indicators (behavioral, physical, and psycholog- ical) as aspects of evidence-based practice in services for persons with intellectual and developmental disabilities (Schalock, Ver- dugo, \& Gomez, 2011). The 5-scale steps of the ICF-CY qualifier from no problem (0) to extreme problem (4) is expected to capture the level of intellectual functioning including borderline func- tioning. It is also expected that the language of functioning will result in significant agreement exists in coding among professio- nals with better agreement among those with more similar back- grounds (e.g., pediatrician vs. psychologist) than those with different backgrounds (e.g., special educator vs. pediatrician or psychologist) is expected.

\section{Specific Aims}

This study examines the utility of the ICF-CYas an alternative form of documentation to the diagnosis and labeling of persons with intellectual disability. Specifically, this study has two aims:

(a) to document the main functional characteristics of function- ing of body structures and body functions in children with intel- lectual disabilities with ICF-CY codes and qualifiers, and (b) to validate the process of coding with interobserver agreement data.

\section{Methods Study Design and Participants}

This observational cross-sectional study was carried out in a Pediatric Neurodevelopment Unit of a tertiary-care pediatric hospital. All the performed procedures were included in the cur- rent clinical practice routine; thus, the children and their families were not subjected to additional interviews or clinical assess- ments for the purpose of this study. The study was approved by the hospital ethics committee and recruitment was dependent on consent by the families to participate in the study.

A sample of 355 children with intellectual disability was drawn from consecutive clinic referrals over a period of 3 years (May 2010-April 2013). The children had a median (min.-max.) age of 3.9 (1.0-17.3) years, with $65.1 \%$ being boys, and all with Portuguese nationality. Inclusion criteria were children with either intellectual disability defined according to the DSM-5 (American Psychiatric Association, 2013) and ICD-10 criteria (WHO, 2007b) or borderline intellectual functioning defined according to the consensus guidelines on borderline intellectual functioning (Salvador-Carulla et al., 2013). Exclusion criteria were children with the diagnoses of autism, cerebral palsy, spe- cific language impairment, hyperactivity, attention-deficit disor- der, and severe birth sensory deficits. 


\section{Measures and Procedures}

The study was conducted in two phases in keeping with the stated aims of documenting functional characteristics of children with intellectual disability and establishing inter-rating agreement of documentation. In the first phase on functional documentation, a neurodevelopmental pediatrician (MCV) collected or updated medical history, made direct observations of the whole sample of 355 children and requested additional investigations, if necessary. Subsequently, these 355 children were evaluated by a clinical psychologist (MJP) who applied two instruments according to chronological age, the Griffiths Mental Developmental Scales (0-8 years) specifically the subscales A-E and F when applicable (Grif- fiths, 1970), and/or the Wechsler Intelligence Scale for Children version III (WISC-III) (Wechsler, 1991) (6-16 years) for subscales of verbal IQ and performance IQ. Derived IQ scores were assigned to established classification levels of borderline functioning ( $<21$ SD) (Salvador-Carulla et al., 2013), mild (<22 SD), mod- erate (<23 SD), severe (<24 SD), and profound (<25 SD) dis- ability (DSM-5) (American Psychiatric Association, 2013). Based on the direct clinical observation and psychological evaluation of each child, the neurodevelopmental pediatrician assigned one of eight ICF-CY codes to observed child characteristics. The eight codes were selected from a checklist of 19 body functions in three domains: global mental functions, specific mental functions, and speech and voice functions, recommended by the Portuguese Ministry of Education and Science (Direçao Geral de Inovaçao e Desenvolvimento Curricular, 2010).

In the second phase on inter-rater agreement, another clinical psychologist (TNM) and a special educator (HR), not involved in the initial observation and evaluation of the 355 children, independently reviewed subsets of the neurodevelopmental observation and psychometric reports in order to assign ICF-CY codes to data. The clinical psychologist and the special educator, also trained in using the ICF-CY, individually reviewed the first consecutive 139 children and 67 children, respectively. Each of the three professionals made their assignments of ICF-CY codes to child data (the neurodevelopmental pediatrician N5355, the clinical psychologist N5139, and the special educator $N$ $567)$ independent of each other. The interobserver agreement among raters was assessed to validate the process of the aforementioned coding by the neurodevelopmental pediatrician.

\section{Statistical Analysis}

The measurement of agreement on a nominal scale-ICF-CY categories—was done by using simple kappa on an ordinal scale. Qualifier values were assigned to each ICF-CY categories-using weighted kappa. A high kappa value provides evidence of inter- rater agreement, whereas a low kappa value suggests greater vari- ability of ratings across raters (Guggenmoos-Holzmann, 1993).

The Cohen's kappa coefficient (Cohen, 1960), expressing the observed proportion of agreement corrected for chance, ranges between 0 and 11. For ratings on an ordinal scale, weighted kappa takes into account the magnitude of impairment values of catego- ries. Disagreement between raters on two 
adjacent categories con- tributes less to the weighted kappa coefficient than disagreement defined by ratings based on the lower and upper extreme category. Kappa values vary between 21 (total disagreement) and 1 (perfect agreement); a value of zero means agreement is due to chance. Kap- pa values were calculated for each of the eight ICF-CY body func- tions and derived values were classified according to robustness of agreement: slight, $0.01<j<0.20$; reasonable, $0.21<j<0.40$; moderate, $0.41<j<$ 0.60; substantial, $0.61<j<0.80$; and almost perfect, $0.81<j<1.00$ (McCartney \& Rosenthal, 2000).

Cohen's kappa coefficient only permits two raters classifying three or more categories. Thus, agreement between the three raters was assessed through the Gwet's first-order agreement coefficient or AC1 statistic and the Gwet's weighted kappa coeffi- cient statistic (Gwet, 2002; Wongpakaran, Wongpakaran, Wed- ding, \& Gwet, 2013), using the AgreeStat (data analysis software system, version 2011.1 www.agreestat.com/agreestat). This was considered a more robust chance-corrected statistic for yielding reliable results, given that the widely used kappa can be mislead- ing in some cases, especially when the sum of the marginal prob- abilities is very different from 1 (Gwet, 2002).

\section{Findings}

The DSM-5 and consensus guidelines for borderline func- tioning supported the classification of intellectual disability level of the 355 children into the following levels: 108 (30.4\%)

borderline level, 153 (43.1\%) mild level, 70 (19.7\%) moderate level, 18 (5.1\%) severe level, and 6 (1.7\%) profound level. Some differ- ences were found in the frequency of borderline and mild intellec- tual capacity among the three raters. In the sample ( $n 5139$ ) rated by the psychologist, $108(77.7 \%)$ were rated at borderline and mild levels, $25(18.0 \%)$ at moderate, $4(2.9 \%)$ at severe, and $2(1.4 \%)$ at profound levels of intellectual disability. In the sample ( $n 567$ ) rat- ed by the special educator, $55(82.1 \%$ ) were rated at borderline and mild levels, $6(8.9 \%)$ at moderate, $4(6.0 \%)$ at severe, and $2(3.0 \%)$ at profound levels of intellectual disability.

A summary of the assignment of ICF-CY codes and qualifiers by the pediatrician for the 355 children is represented in Figure 1.

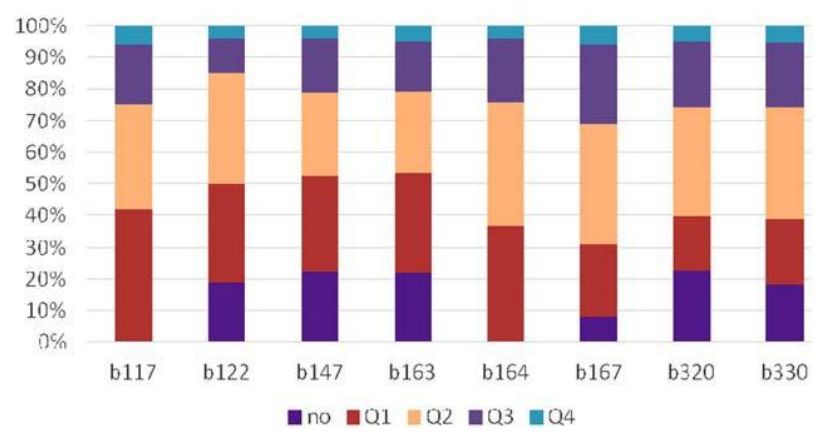


FIGURE 1 - Distribution of qualifier levels (severity) of ICF-CY body functions for 355 with intellectual disabilities (N5355).

The eight ICF-CY body functions coded were b117 intellectual functions, b122 global psychosocial functions, b147 psychomotor functions, b163 basic cognitive functions, b164 higher level cog- nitive functions, b167 language mental functions, b320 articula- tion functions, and b330 fluency and rhythm speech functions. As shown in Figure 1, the most frequent qualifier for codes b117, b147, and b163 was mild (qualifier 1) and for codes b122, b164, b167, b320, and b330, it was moderate (qualifier 2). It is interesting to note that for codes b117 and b164, no child was rated with the qualifier of 0 , meaning the absence of a problem. Across the remaining six codes, the rating of no problem (qualifi- er 0 ), was found for $28-81$ of the 335 children.

For a more detailed analysis of the functional characteristics of children with intellectual disabilities, profiles of ICF-CY codes and qualifiers were produced for each level of intellectual disability. Through this procedure, we analyzed the extent to which the ICF- CYqualifiers were aligned with reference to children's level of intel- lectual disability. As shown in Table 1, qualifier 1 was the most fre- quent value across codes in the borderline functional group (<21 SD), except for codes related to voice and speech (b320 and b330) and qualifier 2 was the most frequent code in mild disability (<22 SD) group. The most frequent qualifier for the moderate dis- ability (<23 SD) group was qualifier 3 except for function b117 (qualifier 2), and qualifier 4 was the most frequent for the groups severe (<24 SD) and profound (<25 SD) disabilities. This analy- sis supports a general correspondence of severity between the ICFCYqualifiers and the levels of intellectual disability.

A comparison of simple kappa for eight body functions rat- ings across by the pediatrician, psychologist, and special educator is summarized in Table 2. Overall, the inter-rater agreement between pediatrician and psychologist ranges from reasonable to moderate for most codes (for codes b320 and b330, this agree- ment is lower). The range of values of the weighted kappa for this pair of raters reveals moderate to substantial agreement, as summarized in Table 3. Similar patterns of inter-rater agreement are found for pediatrician/special educator and psychologist/spe- cial educator pairings. The level of agreement is higher when raters are assigning the qualifiers than when assigning the ICFCY codes. Lower levels of agreement were found between the pediatrician and the special educator. An issue related to the low- er levels of agreement for codes between raters may be differences in the core knowledge of raters related to the conceptual nature of what is being rated. For example, codes b320 and b330 - relat- ed to voice and speech functions-registers the lowest level of agreement between pediatrician/psychologist; code b164-higher level cognitive functions—registers the lowest level of agreement between pediatrician/special educator and between psychologist/ special educators.

Finally, determination of interobserver agreement among the three professionals based on Gwet's AC1 (Table 2) and weighted Gwet's AC1 (Table 3) indicated moderate to near-perfect agree- ment for intellectual functions (code b117), psychomotor func- tions (code b147), basic cognitive functions (code b163); from fair to near perfect agreement for global psychosocial functions (code b122), language mental functions (code b167), and fluency and rhythm speech functions (code b330); and from fair to substantial agreement for higher level cognitive functions (code b164) and articulation functions (code b320). 
TABLE 1 - Profile for each level of intellectual disability, with the ICF-CY qualifiers aligned with the intellectual disability level of reference (in each ICF-CY qualifier, the score more frequently rated according to intellectual level is bold marked)

\begin{tabular}{|c|c|c|c|c|c|c|c|c|c|c|c|c|c|c|c|c|c|c|c|c|c|c|c|c|c|}
\hline \multirow{2}{*}{$\begin{array}{l}\text { Borderline DSM-5 } \\
\mathrm{SD}^{\mathrm{b}}(n 56) \\
\text { ICF-CY qualifiers }\end{array}$} & \multicolumn{5}{|c|}{ Borderline $^{\mathrm{a}}(n 5108)$} & \multicolumn{5}{|c|}{$<22 \mathrm{SD}^{\mathrm{b}}(n 5153)$} & \multicolumn{5}{|c|}{$<23 \mathrm{SD}^{\mathrm{b}}(n 570)$} & \multicolumn{5}{|c|}{$<24 \mathrm{SD}^{\mathrm{b}}(n 518)$} & \multicolumn{5}{|c|}{$<25$} \\
\hline & 0 & 1 & 2 & 3 & 4 & 0 & 1 & 2 & 3 & 4 & 0 & 1 & 2 & 3 & 4 & 0 & 1 & 2 & 3 & 4 & 0 & 1 & 2 & 3 & 4 \\
\hline b117 & 0 & 108 & 0 & 0 & 0 & 0 & 42 & 75 & 35 & 1 & 0 & 0 & 41 & 28 & 1 & 0 & 0 & 0 & 6 & 12 & 0 & 0 & 0 & 0 & 6 \\
\hline b163 & 40 & 68 & 0 & 0 & 0 & 37 & 42 & 56 & 18 & 0 & 0 & 2 & 30 & 34 & 4 & 1 & 0 & 5 & 5 & 7 & 0 & 0 & 0 & 0 & 6 \\
\hline b164 & 0 & 92 & 16 & 0 & 0 & 0 & 35 & 96 & 22 & 0 & 0 & 1 & 28 & 41 & 0 & 0 & 0 & 0 & 8 & 10 & 0 & 0 & 0 & 0 & 6 \\
\hline b167 & 27 & 54 & 27 & 0 & 0 & 1 & 27 & 90 & 32 & 3 & 0 & 0 & 17 & 48 & 5 & 0 & 0 & 0 & 10 & 8 & 0 & 0 & 0 & 0 & 6 \\
\hline b320 & 61 & 34 & 13 & 0 & 2 & 20 & 25 & 91 & 14 & 3 & 0 & 0 & 19 & 51 & 0 & 0 & 0 & 1 & 8 & 9 & 0 & 0 & 0 & 0 & 6 \\
\hline b330 & 61 & 38 & 9 & 0 & 0 & 1 & 28 & 99 & 25 & 0 & 0 & 0 & 22 & 45 & 3 & 0 & 0 & 1 & 8 & 9 & 0 & 0 & 0 & 0 & 6 \\
\hline
\end{tabular}

a Borderline (<21SD) (Salvador-Carulla 2013).

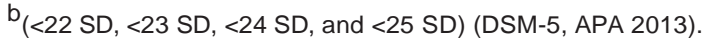

TABLE 2- Interobserver agreement (simple kappa) of 8 body function ratings: Comparison between pediatrician, psychologist, and special educator

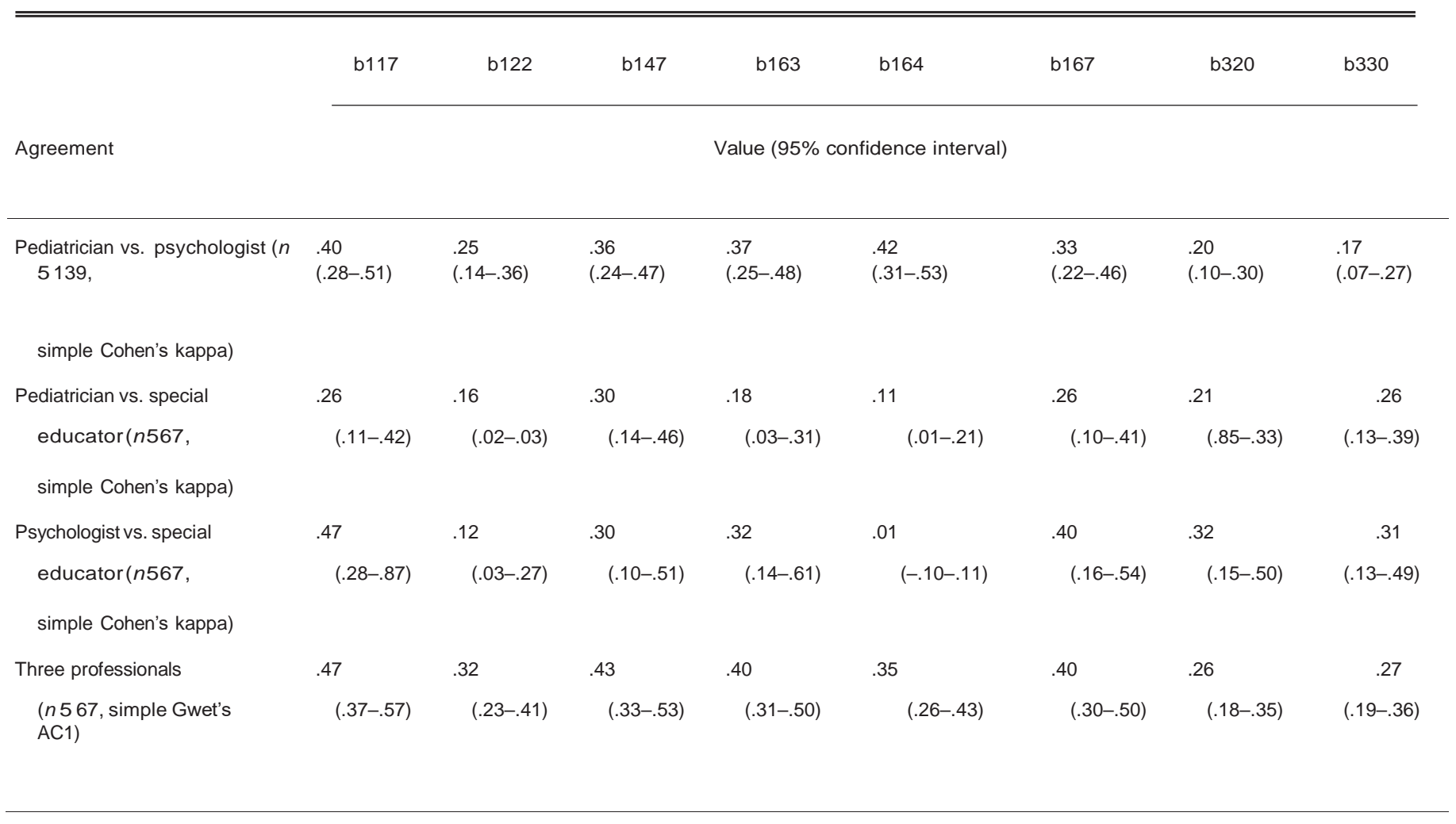




\section{Discussion}

The present findings demonstrate the applicability of the ICF-CY framework and classification system as an alternative approach to the diagnosis of children with intellectual disability using medical or psychiatric classification. This is especially rele- vant with reference to children with borderline intellectual func- tioning, for which application of the ICF-CY was identified as a good practice guideline (Salvador-Carulla et al., 2013). The diag- nostic classifications such as DSM-5 (American Psychiatric Asso- ciation, 2013) and ICD-10 (WHO, 2007b) have limited use to identify, specific educational and social functioning needs for children with borderline functioning. The classification of func- tioning with the ICF-CY was used as the alternative approach to medical diagnosis to determine eligibility in special education in Portugal. In this case, the ICF-CY framework of functioning was adopted for guiding the special education assessment process and the basis for eligibility for special education services (Sanches- Ferreira, Silveira-Maia, \& Alves, 2014).

\section{TABLE 3}

Interobserver agreement (weighted kappa) of 8 body function ratings: Comparison between pediatrician, psychologist, and special educator

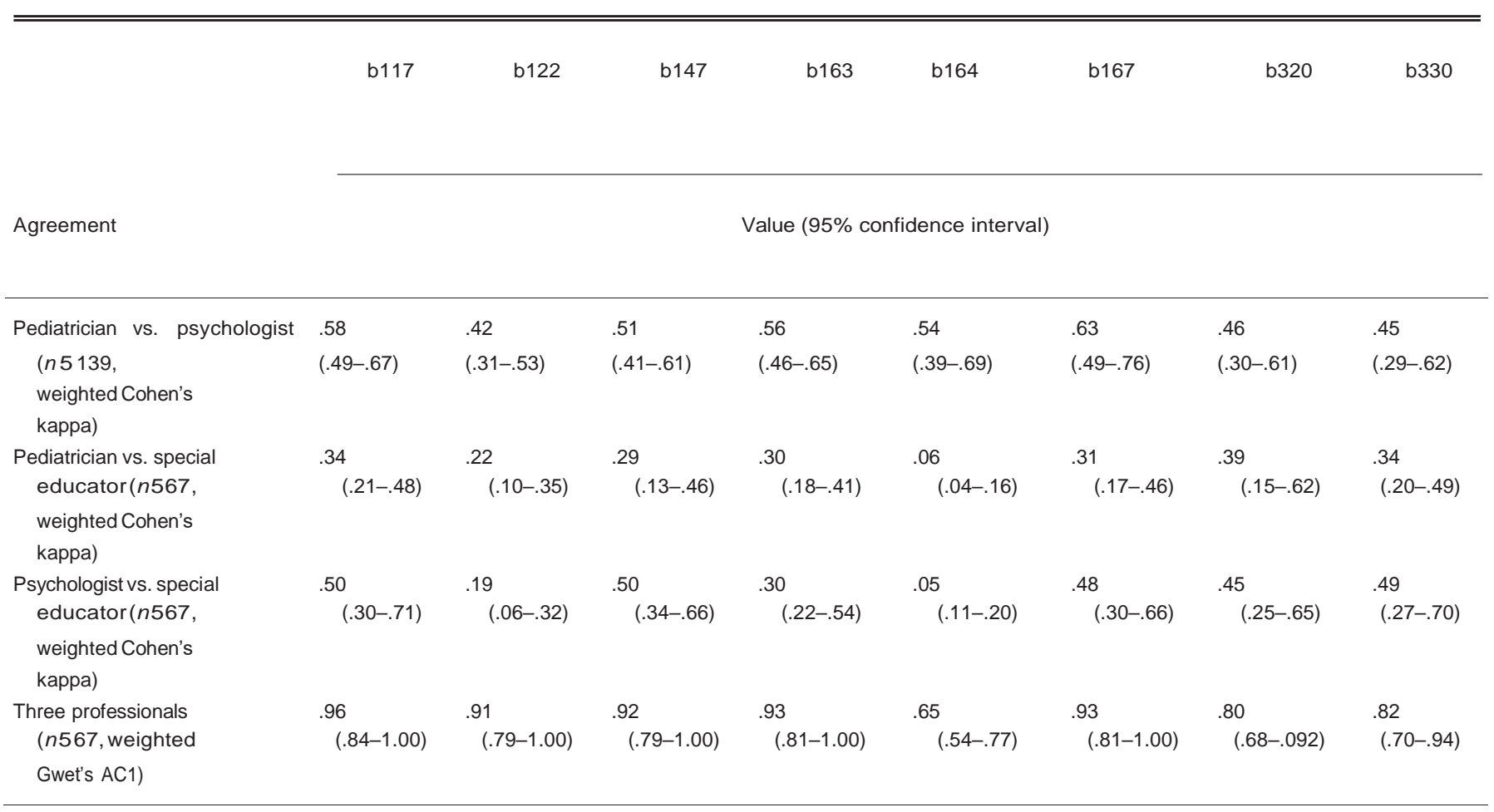


As the ICF-CY model is based on the biopsychosocial approach to disability, it provides a list of alphanumeric codes organized into four main components: body functions, body structures, activities and participation, and environmental factors and a universal qualifier to describe the functional characteristics of children. A critical issue faced by clinicians in application of the ICF-CY in clinical practice and quality management is reli- ability of coding. In keeping with WHO recommendations (Grill et al., 2007), this study calibrated ICF qualifiers on the basis of already existing measures, namely, the Griffiths Mental Scale and WISC-III (Oliveira, Duque, Duarte, Melo, \& Teles, 2012) to eval- uate intellectual capacity of children in Portuguese pediatric units and neurodevelopmental centers.

Although there is a general set of ICF codes disability (Selb et al., 2015), in the absence of a specific set of ICF-CY codes for intellectual disability, a trained neurodevelopmental pediatrician selected a set of ICF-CY codes (WHO, 2007a) within the check- list of body functions recommended by the Portuguese Ministry of Education and Science, considered most relevant to comple- mentary describe the functioning of children with intellectual disability. A set of eight ICF-CY body function codes were used in this study b117 intellectual functions, b122 global psychosocial functions, b147 psychomotor functions, b163 basic cognitive functions, b164 higher level cognitive functions, b167 language mental functions, b320 articulation functions, and b330 fluency and rhythm speech functions. The most frequent qualifier was mild (qualifier 1) for codes b117, b147, and b163 and moderate (qualifier 2) for codes b122, b164, b167, b320, and b330. As expected, the distribution of qualifiers was inversely proportional to the degree of intellectual disability, with mild and moderate qualifier values (1\&2) being more frequent than qualifier values of severe and complete $(3 \& 4)$. Relatively narrow confidence intervals were found for the values assigned to each qualifier.

Profiles of ICF-CY codes and qualifier values for each level of intellectual disability demonstrated the correspondence between the severity of impairments in the selected body functions codes and the respective level of intellectual disability. Specifically, higher frequencies of qualifier value corresponded to level of dis-

ability, qualifier 1 for borderline functioning (<21 SD), qualifier 2 for mild disability ( $<22 \mathrm{SD})$, qualifier 3 for moderate disabili- ty (<23 SD), except for function b117, qualifier 4 for severe (<24 SD), and profound $(<25 \mathrm{SD})$ disabilities. These findings confirmed the congruence between the severity of qualifiers and intellectual disability level, providing a focus for further research to derive a specific core set for persons with intellectual disability. In this regard, core sets of selected ICF categories considered most descriptive of functioning of persons with specific health conditions (Selb et al., 2015) have been identified for conditions such as cerebral palsy (Reed et al., 2005) and neural tube defect (Martinz et al., 2010). To the best of our knowledge, an ICF-CY functional profile for children and adolescents with intellectual disabilities has not yet been described. An ICF-CY core set, espe- cially in the domain of body functions, could serve as a reference to guide assessment of functioning in a standard manner with direct implications for development of congruent intervention plans (Selb et al., 2015).

Interobserver agreement using the ICF-CY body functions codes was assessed in subsamples of 
children and adolescents with intellectual disability between observers with different pro- fessional backgrounds-a neurodevelopmental pediatrician, a clinical psychologist, and a special education teacher. Of children rated by the neurodevelopmental pediatrician, only $39.1 \%$ and $18.9 \%$ children were also rated by the clinical psychologist and the special education teacher, respectively, and this is acknowl- edged as a limitation of the study. The agreement between the three raters was assessed using Gwet statistics (Gwet, 2002). Agreements indicated by Gwet's AC1 and weighted Gwet's AC1 were heterogeneous and higher than Cohen's kappa statistic. Moderate to almost perfect agreements were found for intellectu- al functions, psychomotor functions, and basic cognitive func- tions; fair to almost perfect agreements were found in global psychosocial functions, language mental functions, and fluency and rhythm speech functions; and fair to substantial agreements were found in higher level cognitive functions. An explanation for higher agreements found using the Gwet statistic in relation to Cohen's kappa statistic may be the use of the Gwet's AC1 as a "paradox-resistant" alternative to the unstable kappa coefficient in the situation in which kappa is low despite a high level of agreement (Wongpakaran et al., 2013). In fact, different summa- ry statistics may yield different results for the same data set (Walsh et al., 2014). Given the importance of input from each member of the multidisciplinary team in the description of func- tioning profiles of children and, in turn in the decisionmaking about children' eligibility for special education services, these results have important consequences for practices, enhancing col- laboration between members of multidisciplinary teams. Further research on the utility of this set of codes for describing function- ing profiles of children with intellectual disability is needed to examine interobserver reliability of classification by raters in the same professional category (e.g., pediatricians) and the reliability of classification of other important dimensions of functioning in children with intellectual disability, such activities and participation and related environmental factors.

Professionals involved in the care of children and adolescents with intellectual disability frequently face challenges in designing appropriate educational interventions for students (Raghavendra et al., 2007; Sanches-Ferreira et al., 2013). Lack of a coherent approach for assigning diagnoses and categories may result in inconsistencies in determining eligibility of children with disabil- ities, due to lack of correspondence between diagnoses and the learning and social problems faced by students (SanchesFerreira et al., 2013). In this field, the ICF-based assessment of the nature and severity of disturbances in body functions and structures can serve as the basis for determining eligibility for special education and habilitation, and profiles of functioning (Sanches-Ferreira et al., 2013). The ICF offers a common language in documentation of disability (Simeonsson, 2009) including applications for indi- viduals needing augmentative and alternative communication (Raghavendra et al., 2007). In this study, a set of eight ICF-CY codes was found useful in describing the functioning of children with intellectual disability. Inter-rater agreement of coding was established with the most reliable agreement found for coding intellectual functions (b117) and psychomotor functions (b122).

In summary, a profile of eight ICF-CY body functions codes was found effective in describing the functioning of children with intellectual disability, providing an alternative to medically based classification of 
children based on diagnoses with functionally based classification of children's characteristics. As such, it may also serve as a complementary approach to meet the need for reliable classification of characteristics of children, particularly those in the borderline range of functioning to support professionals in decision-making and provision of intervention for chil- dren with intellectual disabilities in clinical and educational settings. Further research is warranted to confirm our data and define a comprehensive and reliable code set for intellectual dis- ability, reinforcing the validity of ICF-CY in this condition. 


\section{References}

American Psychiatric Association (APA). (2013). Intellectual disability. Diagnostic and statistical manual of mental disorders (5th ed. (DSM-5), pp. 31-41). Washington DC.

Cohen, J. (1960). A coefficient of agreement for nominal scales. Educational and Psychological Measurement, 20, 37-46.

Direçao Geral de Inovaçao e Desenvolvimento Curricular (DGIDC). (2010). Ministerio da Educaçao. Retrieved from: http://www.appdae.

net/documentos/manuais/CIF_Checklist_da_Classificacao_Internacional_da_Funcionalidade.pdf

Griffiths, R. (1970). The abilities of young children: A comprehensive system of mental measurement for the first eight years of life. London: Child Development Research Centre.

Grill, E., Mansmann, U., Cieza, A., \& Stucki, G. (2007). Assessing observer agreement when describing and classifying with the International Classification of Functioning, Disability and Health. Journal of Rehabilitation Medicine, 39, 71-76.

Guggenmoos-Holzmann, I. (1993). How reliable are chance corrected measures of agreement? Statistics in Medicine, 12, 2191-2205.

Gwet, K. (2002). Kappa statistics is not satisfactory for assessing the extent of agreement between raters. Statistical Methods for Inter- Rater Reliability Assessment, 1, 1-5.

Huang, S. W., Lin, L. F., Chou, L. C., Wu, M. J., Liao, C. D., \& Liou, T. H.

(2015). Feasibility of using the international classifi ation of functioning, disability and health core set for evaluation of fall-related risk factors in acute rehabilitation settings. European Journal of Physical and Rehabilitation Medicine, Feb 18. [Epub ahead of print]

Kronk, R. A., Ogonowski, J. A., Rice, C. N., \& Feldman, H. M. (2005).

Reliability in assigning ICF codes to children with special health care needs using a developmentally structured interview. Disability and Rehabilitation, 27, 977-983.

Lee, A. M. (2011). Using the ICF-CY to organize characteristics of children's characteristics. Disability and Rehabilitation, 33, 605-616.

Lollar, D. L., \& Simeonsson, R. J. (2005). Diagnosis to function: Classification for children and youth. Journal of Developmental and Behavioral Pediatrics, 26, 323-330.

Martinz, A., Salghetti, A., Betto, S., Russo, E., Leonardi, M., et al. (2010). The International Classification of Functioning Disability and Health, version for children and youth as a road-map for projecting and programming rehabilitation in a neuropediatric hospital unit. Journal of Rehabilitation Medicine, 42, 4955.

McCartney, K., \& Rosenthal, R. (2000). Effect size, practical importance, and social policy for children. Child Development, 1, 173-180.

Oliveira, G., Duque, F., Duarte, C., Melo, F., Teles, L., et al. (2012). Neurodevelopment pediatrics. National survey on resources and needs. Acta Pediatrica Portuguesa, $43,1-7$. 
Peterson, C., Simeonsson, R. J., Enskar, K., \& Huus, K. (2013). Comparing children's self-report instruments for health-related quality of life using the International Classification of Functioning, Disability and Health for Children and Youth (ICF-CY). Health Quality Life Outcomes, 11, 75-84.

Raghavendra, P., Bornman, J., Granlund, M., \& BjÆrck-Akesson, E. (2007).

The World Health Organization's International Classification of Functioning, Disability and Health: Implications for clinical and research practice in the field of augmentative and alternative communication. Augmentative and Alternative Communication, 23, 349-361.

Reed, G., Lux, J., Bufka, L., Peterson, D., Threats, T., et al. (2005).

Operacionalizing the International Classification of Functioning, Disability and Health. A model to guide clinical thinking, practice and research in the field of cerebral palsy. Seminars in Pediatric Neurology, 11, 5-10.

Salvador-Carulla, L., Garcla-Gutierrez, J. C., Ruiz Gutierrez-Colosia, M.,

Artigas-Pallarès, J., Garcla Ibarrez, J., et al. (2013). Borderline intellectual functioning: consensus and good practice guidelines.

Revista De Psiquiatha Y Salud Mental (Barc.), 6, 109-120.

Sanches-Ferreira, M., Silveira-Maia, M., \& Alves, S. (2014). The use of the International Classification of Functioning, Disability and Health, version for Children and Youth (ICF-CY), in Portuguese special education assessment and eligibility procedures: The professionals' perceptions. European Journal of Special Needs Education, 29, 327-343.

Sanches-Ferreira, M., Simeonsson, R. J., Silveira-Maia, M., Alves, S., Tavares, A., et al. (2013). Portugal's special education law: Implementing the International Classification of Functioning, Disability and Health in policy and practice. Disability and Rehabilitation, 35, 868-873.

Schalock, R. L., Verdugo, M. A., \& Gomez, L. E. (2011). Evidence-based

practices in the field of intellectual and developmental disabilities: an international consensus approach. Evaluation and Program Planning, 34, 273-282.

Selb, M., Escorpizo, R., Kostanjsek, N., Stucki, G., Đstæn, B., et al.

(2015). A guide on how to develop an international classification of functioning, disability and health core set. European Journal of Physical and Rehabilitation Medicine, 51, 105-117.

Simeonsson, R. (2006). Defining and classifying disability in children. In

M.J. Field, A.M. Jette, \& L. Martin, (Eds.) Disability in America: A new look. Washington DC: The National Academies Press.

Simeonsson, R. (2009). ICF-CY: A universal tool for documentation of disability. Journal of Policy and Practice in Intellectual Disabilities, 6, 70-72.

Simeonsson, R., Simeonsson, N. E., \& Hollenweger, J. (2008). International Classification of Functioning, Disability and Health: A common language for special education. In L. Florian, \& M. McLaughlin (Eds.), Disability classification in education. New York: Corwin Publishers. 
Stahl, Y., Granlund, M., Gare-Andersson, B., \& Enskar, K. (2011). Mapping of children's health and development data on population level using the classification system ICF-CY. Scandinavian Journal of Public Health, 39, 51-57.

UN General Assembly, Convention on the Rights of Persons with Disabilities: resolution/adopted by the General Assembly, $\quad 24$ January 2007, A/RES/61/106, available at: http://www.refworld.org/docid/ 45f973632.html [accessed 7 March 2016]

Walsh, P., Thornton, J., Asato, J., Walker, N., McCoy, G., Baal, J., et al. (2014). Approaches to describing inter-rater reliability of the overall clinical appearance of febrile infants and toddlers in the emergency department. PeerJ, 2, e651.

Wechsler, D. (1991). The Wechsler Intelligence Scale for Children (3rd ed.).

San Antonio, TX: The Psychological Corporation.

Wongpakaran, N., Wongpakaran, T., Wedding, D., \& Gwet, K. L. (2013). A comparison of Cohen's kappa and Gwet's AC1 when calculating inter-rater reliability coefficients: A study conducted with personality disorder samples. BMC Medical Research Methodology, 13, 61.

World Health Organization. (2001). International Classification of Functioning, Disability and health: ICF. Geneva:WHO.

World Health Organization. (2007a) International Classification of Functioning, Disability and Health, version for children and youth: ICF-CY. Geneva:WHO.

World Health Organization. (2007b) International Statistical Classification of Diseases and Related Health Problems (10th revision.). Geneva: WHO. 